\title{
Damage diagnosis for offshore fixed wind turbines
}

\author{
D. Agis ${ }^{1}$, Y. Vidal ${ }^{1}$, and F. Pozo ${ }^{1}$ \\ ${ }^{1}$ Mathematics Department, \\ Control, Modeling, Identification and Applications, \\ Escola d'Enginyeria de Barcelona Est \\ ${ }^{1}$ Universitat Politècnica de Catalunya, \\ Campus Diagonal-Besòs, 08019 Barcelona (Spain) \\ Phone number:+0034934137309, e-mail: \{david.agis, yolanda.vidal, francesc.pozo\}@upc.edu
}

\begin{abstract}
This paper proposes a damage diagnosis strategy to detect and classify different type of damages in a laboratory offshore-fixed wind turbine model. The proposed method combines an accelerometer sensor network attached to the structure with a conceived algorithm based on principal component analysis (PCA) with quadratic discriminant analysis (QDA).

The paradigm of structural health monitoring can be undertaken as a pattern recognition problem (comparison between the data collected from the healthy structure and the current structure to diagnose given a known excitation). However, in this work, as the strategy is designed for wind turbines, only the output data from the sensors is used but the excitation is assumed unknown (as in reality is provided by the wind).

The proposed methodology is tested in an experimental laboratory tower modeling an offshore-fixed jacked-type wind turbine. The obtained results show the reliability of the proposed approach.
\end{abstract}

\section{Key words}

Damage diagnosis, structural health monitoring, wind turbine.

\section{Introduction}

Wind energy is one of the best fuel sources since it is clean, relatively cheap and inexhaustible. In order to increase the energy produced by these means, more and more wind farms have been installed in the sea. Taking into account the location of wind turbines and the conditions of the sea, new problems arise, since inspection and maintenance work becomes more difficult. To reduce the enormous logistic and maintenance costs as well as to minimize turbine downtime, wind turbines must be continuously monitored. Among all the monitoring systems, two systems can be highlighted: structural health monitoring (SHM) and condition monitoring $(\mathrm{CM})$. On one hand, a structural health monitoring system verifies the mechanical state of the structure to ensure its proper functioning and determines whether the wind turbine needs some kind of maintenance. On the other hand, a condition monitoring system is able to detect faults in the sensors and/or actuators systems. Traditionally, condition monitoring for WTs has focused on two widely-used methods: vibration analysis and oil monitoring [1]. Therefore, the capability to detect wind turbine damage and faults is crucial to decrease the cost of wind energy [2], [3]. SHM and damage detection have been widely studied in recent years. A review of the state-of-the-art revealed that damage detection is a very active field, but there is not a universal optimum method for it.

This work proposes a complete methodology for damage detection and classification in a laboratory offshore-fixed wind turbine model. The strategy combines: (i) the use of an accelerometer sensor network attached to the structure; (ii) the use of principal component analysis (PCA) as a pre-processing step to both reduce the dimensionality of the data and extract features; and (iii) a quadratic discriminant analysis (QDA). It should be noted that PCA has been widely used in the field of SHM either as the single strategy [4] or in combination with univariate [5] and multivariate [6] hypothesis testing. Furthermore, methods based on principal component analysis (PCA) have also proven its capability to build WT fault detection strategies [7], [3], [8]. However, QDA is most commonly used in medicine [9], [10] and genomics [11], [12], as a classifier or as a pattern-recognition method. A recent application of QDA in WT fault detection is proposed by [13] where an approach for detecting and diagnosing the delamination in wind turbine blades is proposed.

As in [14], it is supposed that the only available excitation of the WTs is the wind turbulence, so the input excitation is assumed to be unknown. Therefore, the scheme of the proposed method can be summarized in the following steps: (i) the wind excitation is simulated as a Gaussian white noise and the data coming from the WT is collected using a set of accelerometers. It is worth remarking that only output data will be used to detect damage; (ii) the raw data is pre-processed using group-scaling to simplify the computation of the principal components; (iii) PCA is selected as a technique to reduce the dimensionality of the data and the computing time of the next step; finally, (iv) the quadratic discriminant analysis (QDA) is used as a classifier. In the end, 10-fold crossvalidation technique 
is employed to estimate the overall accuracy and to avoid over-fitting. In order to validate the proposed approach in this work, the damage detection strategy is applied to different types of predefined damage in a small-scale structure -an experimental laboratory tower modeling an offshore-fixed jacked-type wind turbine-. The results that have been obtained for these predefined damages are included and discussed to demonstrate the reliability of the proposed approach.

The structure of the paper is as follows. We first present the scaled wind turbine model, together with the two types of damage that are introduced at the jacket support and the sensors placed in the tower and jacket. We then present the damage diagnosis strategy that includes how data is collected, reshaped and auto scaled. Subsequently, both PCA and QDA are briefly described. Finally, the main results are summarized and discussed and some conclusions are drawn.

\section{Laboratory Tower Definition}

\section{A. Structure}

The real structure used in this work is a scaled WT tower model, see Figure 1. This structure is $2.7 \mathrm{~m}$ high and it is composed, mainly, of three parts:

1) Jacket support, it is a lattice structure composed with several bars, all of them joined with bolts with a torque of $12 \mathrm{Nm}$.

2) Tower, composed of three sections joined with bolts.

3) Nacelle, modeled in this experiment by the top beam of $1 \mathrm{~m}$ long and $0.6 \mathrm{~m}$ width and a modal shaker located at one edge of the beam.

The shaker simulates the nacelle mass and the environmental effects of the wind over the whole structure. The vibration needed to excite the structure is created by applying an electrical signal to the shaker (Gaussian white noise).

\section{B. Damages}

Two types of damage are introduced at the jacket support:

(a) a $5 \mathrm{~mm}$ crack in one of the bars; and

(b) loosening one of the bolts in the jacket.

Also a healthy replica of the studied bar has been considered. The proposed strategy should be able to detect and classify the studied faults, but also be robust to the replacement of one bar by a new healthy one (avoiding false alarms).

\section{Sensors}

To analyze the structural response, eight triaxial accelerometers are placed in the tower and jacket. The method used to find the optimum location and amount of these sensors is given in [15]. Thus, data from 24 sensors is collected. The nomenclature used for each sensor is given in Table I.

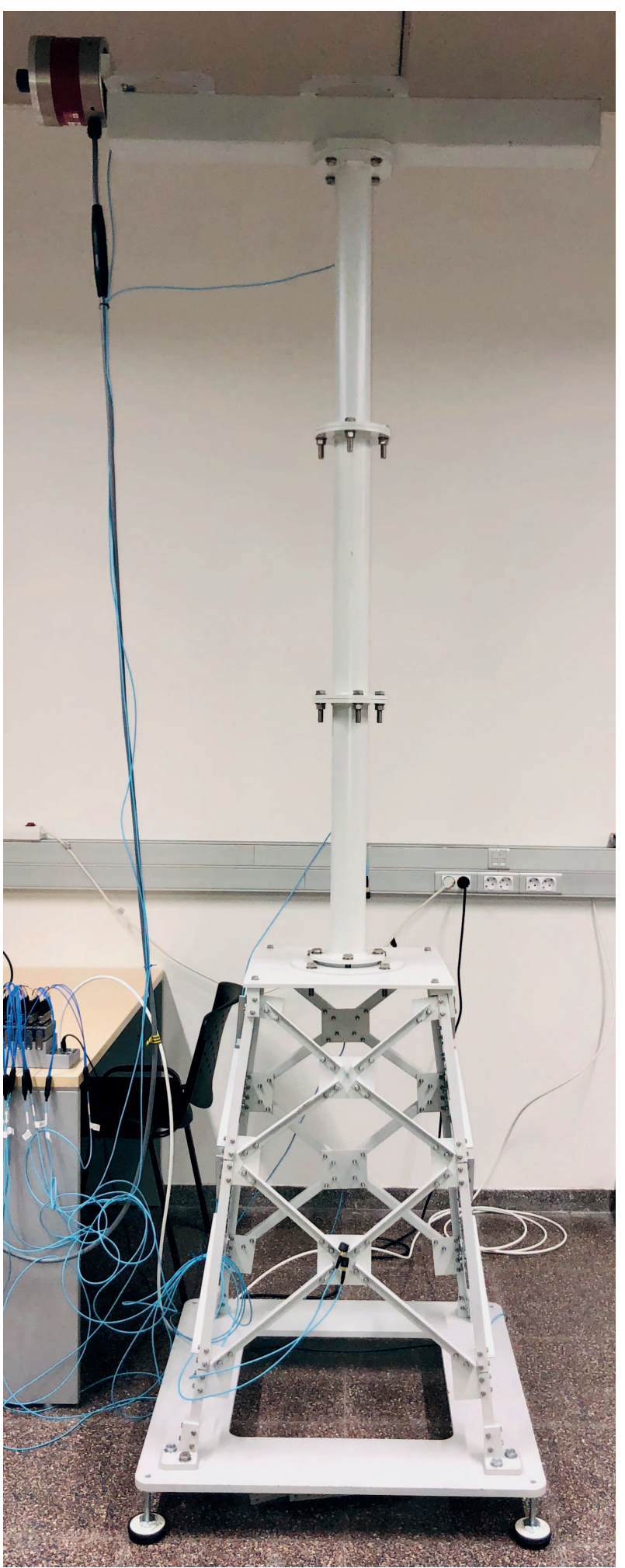

Fig. 1. WT scaled tower model used in the experimental tests (off-shore fixed jacked-type platform).

\section{Damage diagnosis strategy}

\section{A. Data collection}

The time window for each experimental test is $60 \mathrm{sec}-$ onds with a sampling frequency of $1651.6129 \mathrm{~Hz}$. Thus, 
TABLE I

NOMENCLATURE USED TO REFER TO EACH AVAILABLE SENSOR. NOTE THAT $i=1, \ldots, 8$, AS THERE ARE EIGHT ACCELEROMETERS.

\begin{tabular}{ll}
\hline & Sensor \\
\hline$A_{i}^{x}$ & Acceleration in $x$-direction for accelerometer number $i$ \\
$A_{i}^{y}$ & Acceleration in $y$-direction for accelerometer number $i$ \\
$A_{i}^{z}$ & Acceleration in $z$-direction for accelerometer number $i$ \\
\hline
\end{tabular}

each experiment obtains $1651.6129 \times 60=99097$ data measurements from each of the 24 sensors. Given the $k$ th experimental test, the data is initially stored in a matrix $\mathbf{Y}^{(k)} \in \mathcal{M}_{24 \times 99097}(\mathbb{R})$ such that

$$
\mathbf{Y}^{(k)}=\left(\begin{array}{cccc}
y_{1,1}^{(k)} & y_{1,2}^{(k)} & \cdots & y_{1,99097}^{(k)} \\
y_{2,1}^{(k)} & y_{2,2}^{(k)} & \cdots & y_{2,99097}^{(k)} \\
\vdots & \vdots & \ddots & \vdots \\
y_{24,1}^{(k)} & y_{24,2}^{(k)} & \cdots & y_{24,99097}^{(k)}
\end{array}\right)
$$

where the number of rows is given by the number of sensors and the number of columns is equal to the number of time stamps in each experimental test. Note that data in the first row is related to sensor $A_{1}^{x}$, data in the second row is related to sensor $A_{1}^{y}$, third row is related to $A_{1}^{z}$, fourth row to $A_{2}^{x}$, and so on and so forth. Finally, the matrix is reshaped, by concatenating its rows, to form a row-vector with $99097 \times$ $24=2378328$ components. That is, from each experimental test, a row-vector $z^{(k)} \in \mathcal{M}_{1 \times 2378328}(\mathbb{R})$ is obtained such that

$$
z^{(k)}=\left(z_{1}^{(k)}, \ldots, z_{j}^{(k)}, \ldots, z_{24}^{(k)}\right) \in \mathcal{M}_{1 \times(99097 \cdot 24)}(\mathbb{R})
$$

where

$z_{j}^{(k)}=\left(y_{j, 1}^{(k)}, \ldots, y_{j, 99097}^{(k)}\right) \in \mathcal{M}_{1 \times 99097}(\mathbb{R}), j=1, \ldots, 24$.

In this work, a total of 25 experimental tests are conducted. In particular:

(i) 10 tests with the original healthy bar.

(ii) 5 tests with the replica bar.

(iii) 5 tests with the $5 \mathrm{~mm}$ crack damaged bar.

(iv) 5 tests with an unlocked bolt in the jacket.

The data from all the experimental tests is stored in a matrix $\mathbf{Z} \in \mathcal{M}_{25 \times 2378328}$, where each row, $k=1, \ldots, 25$, is given by the row-vector $z^{(k)}$ as defined in Equation (2):

$$
\mathbf{Z}=\left(\begin{array}{c}
z^{(1)} \\
\vdots \\
z^{(k)} \\
\vdots \\
z^{(25)}
\end{array}\right) \in \mathcal{M}_{25 \times(99097 \cdot 24)}(\mathbb{R})
$$

\section{B. Data reshape (Sample Size and Power Analysis)}

The input dataset, $\mathbf{Z}$, consists of a matrix with a small number of experimental tests, only 25 , and a large number of data measurements, $99097 \times 24$. When a small sample size is used in data analysis, this might be insufficient to detect wind turbine damages. For this reason, we propose to reshape the matrix $\mathbf{Z}$ in order to increase the statistical power, by means of increasing the number of experimental tests. In addition, with this reshaping, the time window for each sample is reduced from 60 to 1.46 seconds. Therefore, leading to a fault detection time reduction. Thus, we reshape the matrix $\mathbf{Z}$ as follows: for each row and sensor, we split the 99097 time stamps to 41 subsets of 2417 time instants. Therefore, we get a total of 41 experimental tests with 2417 data measurements for each original row and sensor:

$$
\mathbf{x}_{j}^{(k)}=\left(\begin{array}{ccc}
z_{j, 1}^{(k)} & \cdots & z_{j, 2417}^{(k)} \\
z_{j, 2418}^{(k)} & \cdots & z_{j, 4834}^{(k)} \\
\vdots & \ddots & \vdots \\
z_{j, 96681}^{(k)} & \cdots & z_{j, 99097}^{(k)}
\end{array}\right) \in \mathcal{M}_{41 \times 2417}(\mathbb{R}),
$$

where $z_{j, i}^{(k)}, j=1, \ldots, 24, i=1, \ldots, 99097$ is defined as the $i$-th component of the vector $z_{j}^{(k)}$ defined in Equation (3). Equivalently,

$$
z_{j, i}^{(k)}=y_{j, i}^{(k)}, j=1, \ldots, 24, i=1, \ldots, 99097 .
$$

Similarly, matrix $\mathbf{x}_{j}^{(k)}$ can be defined as

$$
\left(\mathbf{x}_{j}^{(k)}\right)_{m n}=z_{j, 2417 \cdot(m-1)+n}^{(k)}
$$

where $m=1, \ldots, 41$ and $n=1, \ldots, 2417$.

Then, the measurements are arranged in a matrix $\mathbf{X} \in$ $\mathcal{M}_{(41 \cdot 25) \times(2417 \cdot 24)}(\mathbb{R})=\mathcal{M}_{1025 \times 58008}(\mathbb{R})$ :

$$
\mathbf{X}=\left(\begin{array}{ccccc}
\mathbf{x}_{1}^{(1)} & \cdots & \mathbf{x}_{j}^{(1)} & \cdots & \mathbf{x}_{24}^{(1)} \\
\mathbf{x}_{1}^{(2)} & \cdots & \mathbf{x}_{j}^{(2)} & \cdots & \mathbf{x}_{24}^{(2)} \\
\vdots & \ddots & \vdots & \ddots & \vdots \\
\mathbf{x}_{1}^{(25)} & \cdots & \mathbf{x}_{j}^{(25)} & \cdots & \mathbf{x}_{24}^{(25)}
\end{array}\right)
$$

It is to be assumed that larger sample sizes will improve data analysis.

\section{Autoscaling}

The main reason to autoscale the raw data is to simplify the computations for the multiway PCA decomposition. Autoscaling uses column-wise mean-centering followed by division of each column by the standard deviation of that column of matrix $\mathbf{X}$. The result is that each column of the new autoscaled matrix, $\tilde{\mathbf{X}}$, has a mean of zero and a standard deviation of one. The fact that $\tilde{\mathbf{X}}$ is a mean-centered matrix simplifies the empirical covariance matrix computation, needed for the PCA decomposition. 


\section{Principal component analysis and quadratic discriminant analysis}

Recall that, before using a classifier, the data must be processed to obtain the most suitable features. In this work, after the autoscaling step, multiway PCA is selected as the main objective is to reduce computing time for the quadratic discriminant analysis classifier. In this work, only the first 30 components of the PCA decomposition are used as they account for $75 \%$ of the variance. Thus, the transformed coordinates of the $\tilde{\mathbf{X}}$ data in the new basis given by the first 30 principal components are used as features by the QDA strategy.

It is beyond the purpose of this work to give a detailed explanation of the QDA approach. An excellent tutorial about the basic background needed to understand the discriminant analysis classifier is given in [16]. However, it is important to recall that it is assumed that the measurements from each class are normally distributed. Unlike linear discriminant analysis (DA) however, in QDA there is no assumption that the covariance of each of the classes is identical. When the normality assumption is true, the best possible test for the hypothesis that a given measurement is from a given class is the likelihood ratio test. Thus, in a nutshell, the QDA classifier models the likelihood of each class as a Gaussian distribution, and then uses the posterior distributions to estimate the class for a given test point [17]. The Gaussian parameters for each class can be estimated from training points with maximum likelihood estimation. In this work, the one-sample Kolmogorov-Smirnov test, see [18], is used to test the normality of the data. The null hypothesis is that the data comes from a standard normal distribution, against the alternative that it does not come from such a distribution. The test fails to reject the null hypothesis at a $5 \%$ significance level. Furthermore, we confirm the test decision by visually comparing the empirical cumulative distribution function to the standard normal (Gaussian) one, see Figure 2.

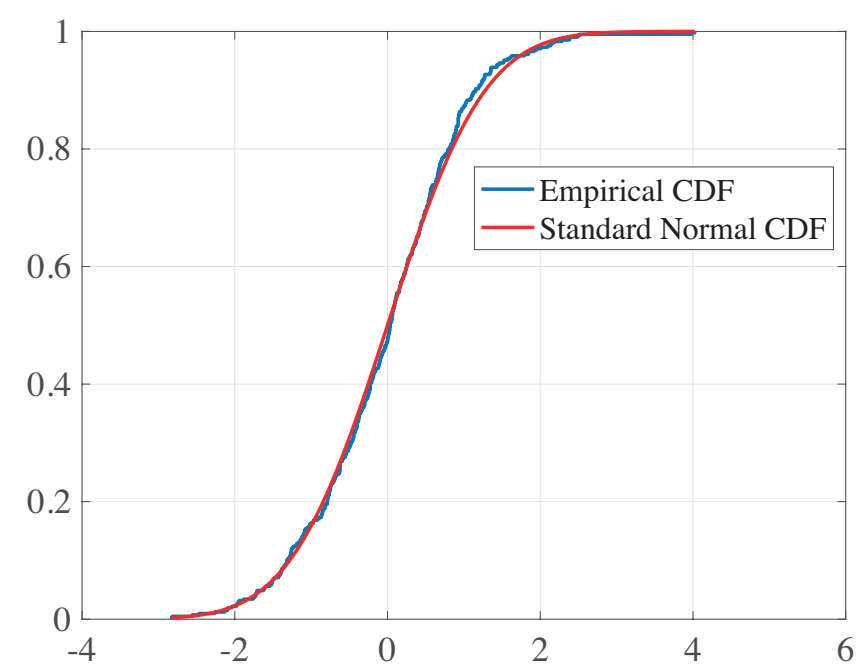

Fig. 2. Empirical cumulative distribution function. has been employed to estimate the overall accuracy and to avoid over-fitting.

\section{Results and discussion}

Table II summarizes the results obtained from the proposed strategy. It presents not only the overall accuracy, but also the training time and prediction speed, as both parameters are critical in real application. Notice that the obtained prediction speed allows this methodology to be deployed for online (real-time) condition monitoring in WTs.

TABLE ॥

SUMMARY OF THE OBTAINED RESULTS.

\begin{tabular}{ll}
\hline Accuracy (\%) & 95.2 \\
Training time (s) & 9.5 \\
Prediction speed (obs/s) & 7300 \\
\hline
\end{tabular}

Besides, a comprehensive decomposition of the error between the true classes and the predicted classes is shown by means of the so called confusion matrix, see Figure 3. In these matrices, each row represents the instances in a true class while each column represents the instances in a predicted class (by the classifier). In particular, first row (and first column) is labeled as 0 and corresponds to the healthy case. Next labels (for rows and columns) correspond to the replica bar (label 1), the $5 \mathrm{~mm}$ crack (label 2), and the unlocked bolt (label 3). From the confusion matrix the following issues can be highlighted. The healthy class has a true positive rate (TPR), that is percentage of correctly classified instances, of $90 \%$ and a false negative rate (FNR), that is percentage of incorrectly classified instances, of $10 \%$. It is noteworthy that the $5 \mathrm{~mm}$ crack damage has a TPR of $99 \%$ and the unlocked bolt damage has a TPR of $100 \%$.

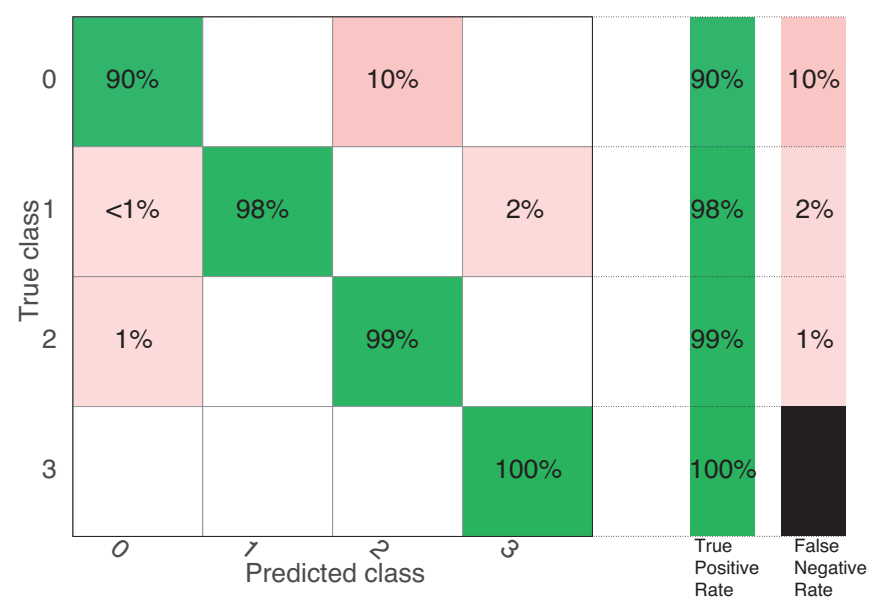

Fig. 3. Confusion matrix.

Finally, in this work, the 10-fold crossvalidation technique 


\section{Conclusions and future work}

This work has proposed a damage detection and classification strategy and tested it in a laboratory WT model. The results show a $95.2 \%$ overall accuracy. The immediate future work is to develop further the proposed strategy for different environmental and operational conditions which could be modeled by using different amplitudes for the white noise excitation.

\section{Acknowledgement}

This work has been partially funded by the Spanish Ministerio de Economía, Industria y Competitividad (MINECO) through the research project DPI2014-58427-C2-1-R; by the Spanish Agencia Estatal de Investigación (AEI) - Ministerio de Economía, Industria y Competitividad (MINECO), and the Fondo Europeo de Desarrollo Regional (FEDER) through the research project DPI2017-82930-C2-1-R; and by the Generalitat de Catalunya through the research project 2017 SGR 388. We gratefully acknowledge the support of NVIDIA Corporation with the donation of the Titan Xp GPU used for this research. We thank the Universitat Politècnica de Catalunya (UPC) for pre-doctoral fellowship (to D. A.).

\section{References}

[1] P. Tchakoua, R. Wamkeue, M. Ouhrouche, F. Slaoui-Hasnaoui, T. A. Tameghe, and G. Ekemb, "Wind turbine condition monitoring: Stateof-the-art review, new trends, and future challenges," Energies, vol. 7, no. 4, pp. 2595-2630, 2014.

[2] F. Pozo, Y. Vidal, and Ó. Salgado, "Wind turbine condition monitoring strategy through multiway pca and multivariate inference," Energies, vol. 11, no. 4, p. 749, 2018.

[3] F. Pozo and Y. Vidal, "Damage and fault detection of structures using principal component analysis and hypothesis testing," in Advances in Principal Component Analysis, pp. 137-191, Springer, 2018.

[4] L. Mujica, J. Rodellar, A. Fernandez, and A. Güemes, "Q-statistic and t2-statistic pca-based measures for damage assessment in structures," Structural Health Monitoring, vol. 10, no. 5, pp. 539-553, 2011.

[5] L. Mujica, M. Ruiz, F. Pozo, J. Rodellar, and A. Güemes, "A structural damage detection indicator based on principal component analysis and statistical hypothesis testing," Smart materials and structures, vol. 23, no. 2, p. 025014, 2013.

[6] F. Pozo, I. Arruga, L. E. Mujica, M. Ruiz, and E. Podivilova, "Detection of structural changes through principal component analysis and multivariate statistical inference," Structural Health Monitoring, vol. 15, no. 2, pp. 127-142, 2016.

[7] Y. Wang, X. Ma, and P. Qian, "Wind turbine fault detection and identification through pca-based optimal variable selection," IEEE Transactions on Sustainable Energy, 2018.

[8] P. F. Odgaard and J. Stoustrup, "Gear-box fault detection using timefrequency based methods," Annual Reviews in Control, vol. 40, pp. 50$58,2015$.

[9] R. S. Ryback, M. J. Eckardt, R. R. Rawlings, and L. S. Rosenthal, "Quadratic discriminant analysis as an aid to interpretive reporting of clinical laboratory tests," Jama, vol. 248, no. 18, pp. 2342-2345, 1982.

[10] G. Zonta, G. Anania, B. Fabbri, A. Gaiardo, S. Gherardi, A. Giberti, N. Landini, C. Malagù, L. Scagliarini, and V. Guidi, "Preventive screening of colorectal cancer with a device based on chemoresistive sensors," Sensors and Actuators B: Chemical, vol. 238, pp. 1098-1101, 2017.

[11] M. Q. Zhang, "Identification of protein coding regions in the human genome by quadratic discriminant analysis," Proceedings of the National Academy of Sciences, vol. 94, no. 2, pp. 565-568, 1997.

[12] L. Zhang and L. Luo, "Splice site prediction with quadratic discriminant analysis using diversity measure," Nucleic Acids Research, vol. 31 , no. 21 , pp. 6214-6220, 2003.
[13] A. Arcos Jiménez, C. Q. Gómez Muñoz, and F. P. García Márquez, "Machine learning for wind turbine blades maintenance management," Energies, vol. 11, no. 1, p. 13, 2017.

[14] F. Pozo and Y. Vidal, "Wind turbine fault detection through principal component analysis and statistical hypothesis testing," Energies, vol. 9, no. 1 , p. 3, 2015.

[15] E. Zugasti Uriguen, Design and validation of a methodology for wind energy structures health monitoring. $\mathrm{PhD}$ thesis, Universitat Politècnica de Catalunya, 2014.

[16] A. Tharwat, "Linear vs. quadratic discriminant analysis classifier: a tutorial," vol. 3, p. 145, 012016.

[17] J. Friedman, T. Hastie, and R. Tibshirani, The elements of statistical learning, vol. 1. Springer series in statistics New York, NY, USA:, 2001.

[18] F. J. Massey Jr, "The kolmogorov-smirnov test for goodness of fit," Journal of the American statistical Association, vol. 46, no. 253, pp. 68-78, 1951. 